\section{European Centre for Multidisciplinary Research on Natural Disasters}

Orožen, Adamič Milan, Slovene Academy of Sciences and Arts, Research Centre, The Anton Melik Institute of Geography, Novi Trg 5, 61000 Ljubljana, Slovenia

Located between West and East, North and South, Slovenia is a country of extremely varied landscapes at the intersection of several main European macro-geographical units: the Alps, the Mediterranean, the Pannonian basin. Slovenia therefore encounters a variety of natural phenomena, a wide palette of natural disasters and threats, and is therefore an appropriate focus point for research of this kind.

In Slovenia we have considerable experience in the stated orientation of the proposed Centre. Our journal UJMA - to which a wide variety of experts from the most varied fields contribute demonstrates this most concretely. The basic aims of UJMA and the research initiatives around it are identical in many ways to the orientation set out for the "International Decade for Natural Disaster Reduction." We propose the establishment of a similar journal in English for the purpose of dealing with these issues in the European sphere as one of the first tasks of the Centre. A journal of natural disasters would be an important medium for linking disciplines, research, and endeavours in various countries. At present, there is no similar journal published in Europa.

The documents of the OPA (Open Partial Agreement on the Prevention of, Protection Against, and Organization of Relief in Major Natural and Technological Disasters, Resolution /87/2) especially emphasize that the aim of the group is the multidisciplinary study of cooperative methods for the prevention of, protection against, and organization of relief after major natural and technological disasters. From a review of activities undertaken within the framework of the OPA, it is evident that a centre such as we propose doesn't exist in Europe at present. Given the tasks set out by OPA and United Nations documents, the necessity to estabish such a cente is clear.

A concentrated global effort to reduce the destructive impact of natural disasters was launched on December 11, 1989, when the United Nations General Assembly proclaimed the "International Decade for Natural Disaster Reduction" (IDNDR) beginning January 1, 1990. Preparation for IDNDR began in 1988 with General Assembly Resolution $42 / 169$ in which the everall objective of IDNDR was defined: to reduce the loss of life, property damage, and economic and social disruption caused by natural disasters, including among others the following specific goals:

- to devise appropriate guidelines and strategies for the appication of existing scientific and technical knowledge, taking into account the cultural and economic diversity among nations;

- to foster scientific and engineering endeavours aimed at closing critical gaps in knowledge in order to reduce loss of life and property;

- to disseminante existing and new technical information related to measures for the assessment, prediction, and mitigation of natural disasters.

Several mechanisms for worldwide participation have been created, emphasizing scientific and professional institutions which are well equipped to contribute to IDNDR by promoting the application of existing knowledge to disasters of all types in widely differing communities and by fostering endeavours to mitigate hazard and their impacts.

The main goal of the Centre for Multidisciplinary Research on Natural Disasters is to encourage a multidisciplinary approach to reserach on natural disasters and their consequences. During natural disasters and their aftermath, the central problem is the linkage-correlation between man and the natural phenomenon. In the explanation of causes and the study of natural disasters, the man-environmental relationship is an essential stepping stone in solving the problem. It is an economic question, a question of survival, development, and the future.

Previous experience has shown that possession of the widest possible information on natural disasters has essential significance for the successful solution of problems, the establishment of appropriate measures, and prevention. In many cases today, this knowledge is scattered, uncorrelated, insufficient, or even unpublished, and is often ignored or unused. Therefore, the basic task of the Centre is to contribute to understanding natural disasters through multidisciplinary research and with it to the improvement of preventive activity, to mitigating the impact of disasters, and to reducing loss of life and property. For qualitative progress, coordination and cooperation among researchers, research groups, and various disciplines is necessary.

We must consider the entire character of natural disasters. Reactions to the occurrence of natural disasters are mainly oriented toward post-disaster operations, ie "putting out the fire", and not enough attention is given research and other endeavours to study such disasters in order to take more effective measures.

The goal of the Centre is to stimulate the multidisciplinary study of natural disasters through the following perspectives and methods:

- case studies,

- natural disasters as natural processes,

- environmental and social effects of natural disasters,

- responses to natural disasters,

- development of research methodologies,

- organization of research seminars and conferences,

- publication and dissemination of research results.

The primary task of the Centre is research and coordination of research during major natural disasters, the upgrading of information on natural disasters, and the formation of multidisciplinary research groups for the elaboration of complex threat assessments which should contribute to more effective protection from the consequences of natural disasters.

It would be sensible to utilize existing knowledge, current work, and successful research in various countries, to link interested parties with the help of the Centre, and to contribute to the improvement of research methods.

Here is the idea and some initial effortshas been done. We do invite institutions and people to help us in its creation and realization.

Existing training and research centres which are undertaken via Partial Agreement Network in Europe:

1. the European Centre for Disaster-Related Medicine, San Marino (RSM);

2. the European University Centre for the Cultural Heritage Ravello (I);

3. the European Training Centre for Natural Disasters, Ankara (TR);

4. the European Centre on Prevention and Forecasting of Earthquakes, Athens (GR);

5. the European Centre of Geodynamics and Sismology, Luxembourg

6. the European Mediterranean Seismological Centre, Strasbourg (F);

7. the Euro-Mediterranean Centre on Marine Contamination Hazards, La Valleta (Malta);

8. the European Research Centre on Information in the Event of Disaster Situations, Madrid (E).

Location of the Centres in Europe: No Centre in Eastern Europe! 